\title{
Light-Dependent Translocation of Arrestin in the Absence of Rhodopsin Phosphorylation and Transducin Signaling
}

\author{
Ana Mendez, ${ }^{1}$ Janis Lem, ${ }^{4}$ Melvin Simon, ${ }^{5}$ and Jeannie Chen ${ }^{1,2,3}$ \\ ${ }^{1}$ Zilkha Neurogenetic Institute, The Mary D. Allen Laboratory for Vision Research, Beckman Macular Research Center, Doheny Eye Institute and \\ Departments of ${ }^{2}$ pphthalmology and ${ }^{3}$ Cell and Neurobiology, Keck School of Medicine of the University of Southern California, Los Angeles, California \\ 90089, ${ }^{4}$ Department of Ophthalmology, Molecular Cardiology Research Institute and Program in Genetics, New England Medical Center and Tufts \\ University School of Medicine, Boston, Massachusetts 02111, and ${ }^{5}$ Division of Biology, California Institute of Technology, Pasadena, California 91125
}

Visual arrestin plays a crucial role in the termination of the light response in vertebrate photoreceptors by binding selectively to lightactivated, phosphorylated rhodopsin. Arrestin localizes predominantly to the inner segments and perinuclear region of dark-adapted rod photoreceptors, whereas light induces redistribution of arrestin to the rod outer segments. The mechanism by which arrestin redistributes in response to light is not known, but it is thought to be associated with the ability of arrestin to bind photolyzed, phosphorylated rhodopsin in the outer segment. In this study, we show that light-driven translocation of arrestin is unaffected in two different mouse models in which rhodopsin phosphorylation is lacking. We further show that arrestin movement is initiated by rhodopsin but does not require transducin signaling. These results exclude passive diffusion and point toward active transport as the mechanism for lightdependent arrestin movement in rod photoreceptor cells.

Key words: arrestin; transducin; RPE65; rhodopsin phosphorylation; rod photoreceptor; retina

\section{Introduction}

Vertebrate rods are highly compartmentalized neurons in the retina specialized in the conversion of photons into neural signals. Phototransduction takes place at the rod outer segment compartment, which is separated by a thin, nonmotile cilium from the rod inner segment, the site for metabolism and protein synthesis.

Light initiates phototransduction by activating rhodopsin, which then transmits and amplifies the signal by activating the visual G-protein transducin (Tr) (Burns and Baylor, 2001; Arshavsky et al., 2002). Termination of the light signal requires that light-activated rhodopsin is deactivated by incorporation of multiple phosphates at its $\mathrm{C}$ terminus by rhodopsin kinase (RK) (Wilden et al., 1986; Chen et al., 1999a; Mendez et al., 2000) and the subsequent binding of arrestin (Arr). High-affinity arrestin binding to phosphorylated photolyzed rhodopsin prevents its further interaction with transducin (Wilden et al., 1986; Wilden 1995; Xu et al., 1997).

Rod cells are constantly exposed to different lighting environments and show a remarkable plasticity in adapting to new light-

Received Nov. 13, 2002; revised Jan. 31, 2003; accepted Feb. 4, 2003.

This work was supported by National Institutes of Health (NIH) Grants EY12155, EY12703 (J.C.), EY12008, and AG12288 (M.S.), NIH Specialized Centers of Research in Ischemic Heart Disease (J.L.), the Beckman Macular Research Center (J.C.), and the Massachusetts Lions Eye Research Fund (J.L.). J.C. is a Research to Prevent Blindness James S. Adams Scholar and a Beckman Investigator. We thank Dr. N. Rao for providing the arrestin antibody and the Specialized Imaging Core of the Doheny Eye Institute (National Eye Institute Grant EY03040) for their technical support and guidance.

Correspondence should be addressed to Jeannie Chen, Zilkha Neurogenetic Institute, Departments of Ophthalmology and Cell and Neurobiology, Keck School of Medicine of the University of Southern California, 1501 San Pablo Street, ZNI 223, Los Angeles, CA 90089-2821. E-mail: jeannie@usc.edu.

Copyright $\odot 2003$ Society for Neuroscience $\quad 0270-6474 / 03 / 233124-06 \$ 15.00 / 0$ ing conditions by using multiple regulatory mechanisms (Koutalos and Yau, 1996; Pugh et al., 1999; Burns and Baylor, 2001; Fain et al., 2001). One mechanism that may contribute to regulation of the performance of the rod cell is the light-driven redistribution of certain signal-transducing proteins within the compartments of the rod cell (Sokolov et al., 2002). Both transducin and arrestin immunoreactivity redistribute in rods in response to light (Broekhuyse et al., 1985, 1987; Philp et al., 1987; Mangini and Pepperberg, 1988; Whelan and McGinnis, 1988). Arrestin immunostaining predominates at rod inner segments, the outer nuclear layer, and the outer plexiform layer of dark-adapted retinas, shifting to rod outer segments during light exposure. Immunoreactivity of the transducin $\alpha$ and $\beta$ subunits shifts in the opposite direction in response to light (Philp et al., 1987; Whelan and McGinnis, 1988; McGinnis et al., 1992). Recently, Sokolov et al. (2002) confirmed the physiological movement of transducin by combining serial tangential cryosectioning of the retina with Western blot analysis and demonstrated that this movement extends the range of light intensities in which the rods can operate. The light-driven redistribution of arrestin was recently reexamined by Peterson et al. (2003), who expressed an arrestin-green fluorescent protein (GFP) fusion protein in transgenic Xenopus and observed light-dependent translocation of GFP fluorescence to rod outer segments. This study confirmed that the arrestin immunolocalization studies reflected genuine arrestin redistribution rather than an epitope-masking artifact.

What is the mechanism behind light-triggered arrestin movement in photoreceptor cells? One straightforward hypothesis is that light causes the depletion of free arrestin at rod outer segments by recruiting it to the disk membranes during phosphorylation of photolyzed rhodopsin (Wilden et al., 1986). The result- 
ing gradient of free arrestin would then promote its diffusion from proximal compartments to rod outer segments. In support of this notion, the incubation of retinas with hydroxylamine, which inhibits the phosphorylation of photoactivated rhodopsin, was reported to decrease the extent of arrestin translocation during light exposure (Mangini et al., 1994).

Herein, we use a genetic approach to explore whether generation of photoactivated, phosphorylated rhodopsin and/or transducin signaling is responsible for light-dependent arrestin redistribution. We show that arrestin translocation is not affected in two mouse models deficient in rhodopsin phosphorylation, nor in mice lacking transducin. We also provide evidence that light-driven arrestin translocation is mediated by rhodopsin but that the mechanism is independent of transducin signaling.
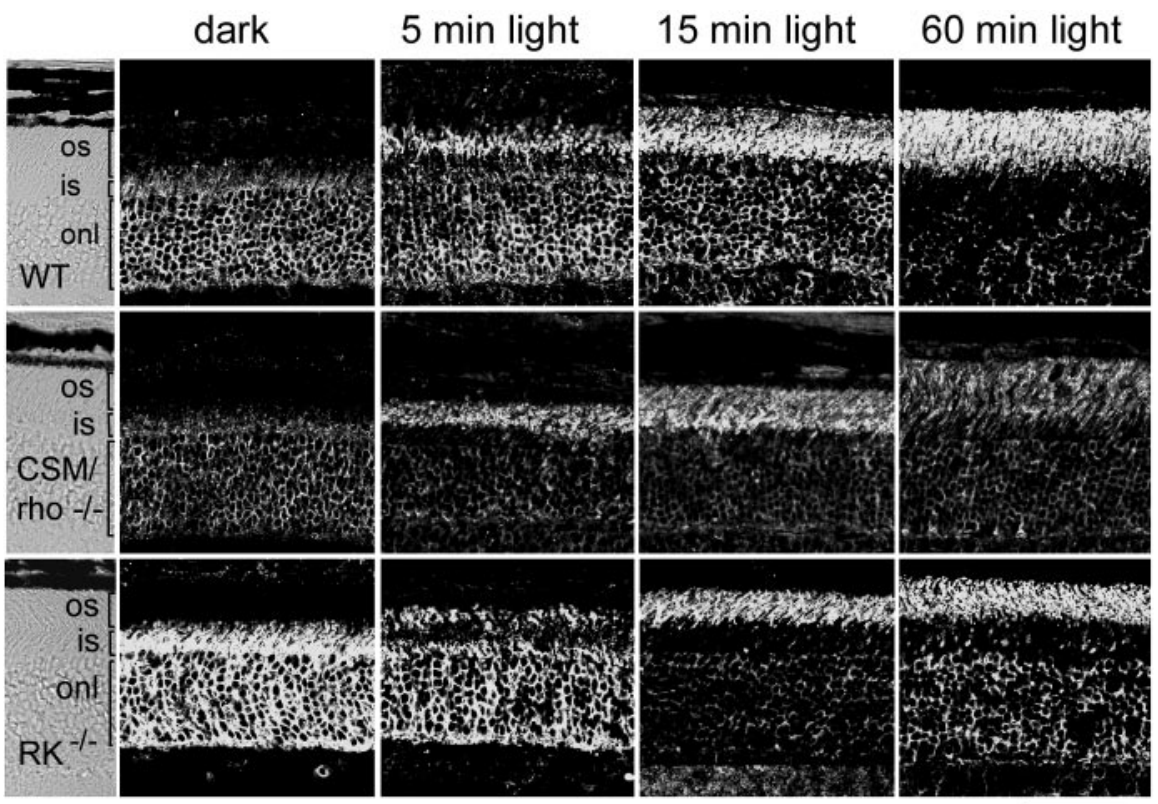

$70 \mu \mathrm{m}$

\section{Materials and Methods}

Mice. All experimental procedures were performed in compliance with National Institutes of Health guidelines and the Society for Neuroscience Policy on the Use of Animals in Neuroscience Research. CSM/rho ${ }^{-1-}$ mice (rhodopsin knock-out mice expressing a mutant rhodopsin in which all of the phosphorylation sites at the $\mathrm{C}$ terminus were substituted to $\mathrm{Ala}$, or "completely substituted mutant" rhodopsin) were derived at the University of Southern California as described previously (Mendez et al., 2000); $\mathrm{RK}^{-1-}$ and $\mathrm{Arr}^{-1-}$ mice were derived at the California Institute of Technology on a C57BL/6 × 129/SvJ background (Xu et al., 1997; Chen et al., 1999a). $\operatorname{Tr}_{\alpha}{ }^{-1-}$ mice were derived at Tufts University on a $\mathrm{BALB} / \mathrm{c} \times 129 / \mathrm{SvJ}$ background (Calvert et al., 2000). Mice lacking retinal pigment epithelium 65 (RPE65 ${ }^{-1-}$ mice) were kindly provided by Dr. Redmond [National Institutes of Health, Bethesda, MD (Redmond et al., 1998)]. Wild-type controls were obtained from breeding pairs established with C57BL/6-DBA F1s.

Light exposure. Experiments were performed on mice at 1 month of age, before the onset of retinal degeneration in $\mathrm{CSM} / \mathrm{rho}^{-1-}$ and RPE65 ${ }^{-1-}$ mice. The CSM/rho ${ }^{-1-}$ and $\mathrm{RK}^{-1-}$ mice, as well as the $\mathrm{Arr}^{-1-}$ and the control mice, were born and housed under constant darkness. $\operatorname{Tr}_{\alpha}{ }^{-1-}, \operatorname{Tr}_{\alpha}{ }^{-1-} / \mathrm{RK}^{-1-}$, and RPE65 ${ }^{-1-}$ mice were born and maintained under $12 \mathrm{hr}$ light/dark cycles and were dark-adapted for at least $12 \mathrm{hr}$ before the analysis. For the dark condition, animals were killed, and the eyes were enucleated and fixed for immunocytochemical analysis under infrared illumination. For the light condition, mice were exposed to diffuse white light (2000 lux intensity at cage level) for the indicated times after pupils were dilated with $0.5 \%$ tropicamide and $2.5 \%$ phenylephrine hydrochloride.

Immunocytochemistry. Eyecups were fixed in $0.1 \mathrm{M}$ cacodylate buffer, $\mathrm{pH} 7.2$, containing $4 \%$ paraformaldehyde and $0.5 \%$ glutaraldehyde for 3 $\mathrm{hr}$ at room temperature, washed, and cryoprotected for $12 \mathrm{hr}$ in $0.1 \mathrm{M}$ cacodylate buffer, $\mathrm{pH} 7.2$, containing $30 \%$ sucrose at $4^{\circ} \mathrm{C}$. Eyecups were then embedded in O.C.T. (Tissue-Tek, Sakura Finetech, Torrance, CA) and sectioned at $-18^{\circ} \mathrm{C}$. Ten micrometer sections were collected and incubated in blocking solution (PBS containing 1\% BSA, 5\% normal goat serum, and $0.3 \%$ Triton $\mathrm{X}-100)$ for $1 \mathrm{hr}$ at room temperature. Sections were incubated with primary antibody diluted in PBS, with $1 \%$ BSA, $1 \%$ normal goat serum, and $0.1 \%$ Triton X-100 for either $2 \mathrm{hr}$ at room temperature or overnight at $4^{\circ} \mathrm{C}$. For arrestin detection, a polyclonal antibody raised against purified bovine arrestin was used (a generous gift from N. Rao, University of Southern California). To detect $\operatorname{Tr}_{\alpha}$, a rabbit polyclonal antibody made against residues $85-103$ of $\operatorname{Tr}_{\alpha}$ was used (Raport et al., 1994). Proteins were visualized with FITCconjugated goat anti-rabbit IgG (Vector Laboratories, Burlingame, CA), and images were acquired on an LSM 510 Zeiss (Thornwood, NY) laser scanning confocal microscope. To compare the signal between different mouse models, all images in each individual experiment were acquired with a fixed detection gain.

\section{Results}

Light-dependent arrestin movement may be signaled through two well characterized biochemical events: generation of photolyzed, phosphorylated rhodopsin and activation of transducin. Light-activated, phosphorylated rhodopsin is a high-affinity binding target for arrestin (Wilden et al., 1986), and depletion of arrestin in the outer segment compartment as it is recruited to the disk membranes may create a concentration gradient for diffusion of soluble arrestin toward the outer segment. On the other hand, photolyzed rhodopsin may signal arrestin movement through molecular motors in a process mediated by transducin signaling and/or transducin translocation. To determine whether these events underlie arrestin movement, we examined arrestin localization in mice defective in rhodopsin phosphorylation (Chen et al., 1999a; Mendez et al., 2000) and subsequently in mice lacking transducin (Calvert et al., 2000). To confirm that lightdependent arrestin movement is signaled through rhodopsin, we examined arrestin localization in retinas from RPE65 $5^{-1-}$ mice that are deficient in rhodopsin because of a defect in the visual cycle (Redmond et al., 1998).

\section{Light causes arrestin translocation in the absence of rhodopsin phosphorylation}

To determine whether rhodopsin phosphorylation is the driving force behind light-triggered arrestin movement from the inner to the 


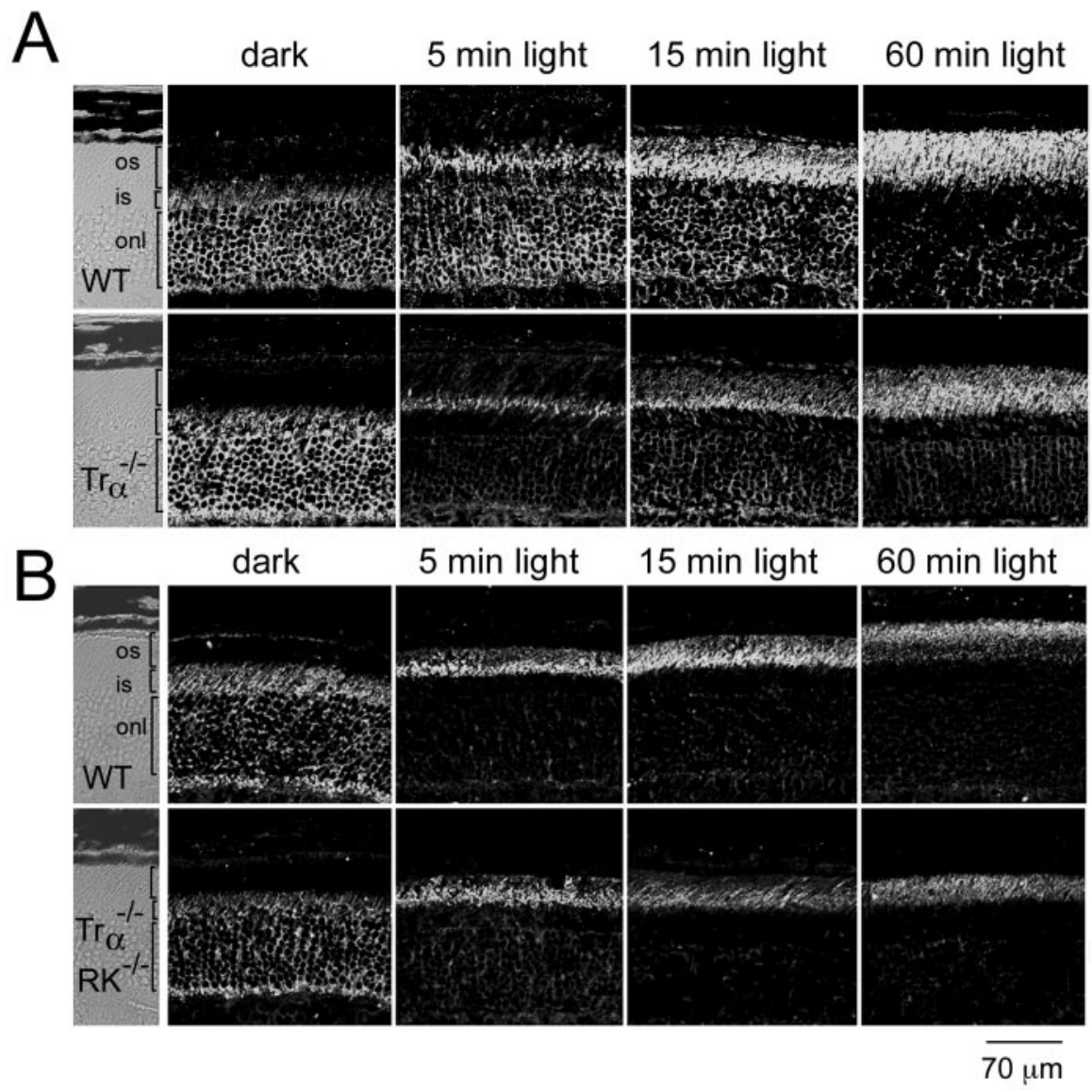

Figure 2. Time course of light-driven arrestin translocation in $\operatorname{Tr}_{\alpha}{ }^{-1-}$ retinas $(A)$ and in $\operatorname{Tr}_{\alpha}{ }^{-1-} / \mathrm{RK}^{-1-}$ retinas $(B)$. The light-exposure protocol was the same as in Figure 1. Wild-type (WT) mice in $A$ and $B$ were processed in parallel to $\operatorname{Tr}_{\alpha}{ }^{-1-}$ and $\mathrm{Tr}_{\alpha}{ }^{-1-} / \mathrm{RK}^{-1-}$ mice, respectively, under identical conditions of light exposure and immunocytochemical reactions. Arrestin immunoreactivity shifts from the outer nuclei and inner segments of rods toward the rod outer segments to a similar extent and with approximately the same time course in the presence or absence of $\operatorname{Tr}_{\alpha}$ and RK. os, Outer segment; is, inner segment; onl, outer nuclear layer.

outer segment of rods in the intact retina, the immunolocalization of arrestin was analyzed in two mouse models that do not exhibit lightdependent rhodopsin phosphorylation: $\mathrm{CSM} / \mathrm{rho}^{-1-}$ mice (Mendez et al., 2000) (see Materials and Methods) and $\mathrm{RK}^{-/-}$mice (Chen et al., 1999a).

Arrestin immunolocalization was assayed in retinal sections from dark-adapted mice or mice exposed to 2000 lux diffuse fluorescent light for different time periods after dark adaptation (Fig. 1). Because all transgenic lines were in a pigmented background $(129 / \mathrm{sv} \times \mathrm{C} 57 \mathrm{BL} / 6)$, pupils from all mice were dilated to maximize the amount of light that reaches the retina. As can be seen in the dark-adapted wild-type retinas, arrestin localizes predominantly to the cytoplasmic space that includes the perinuclear region, the inner segment, and the synaptic terminal of rods (Fig. 1, dark). During light exposure, arrestin immunoreactivity first accumulates in the proximal region of the outer segment (Fig. 1, 5 min light) and continues to extend toward the distal tip of the outer segment as the signal decreases from the cytoplasmic compartment (Fig. 1, $15 \mathrm{~min}, 60 \mathrm{~min}$ light). This pattern of arrestin immunoreactivity and the time course of arrestin translocation were similar to previous reports (Philp et al., 1987; Mangini and Pepperberg, 1988; Whelan and McGinnis, 1988; McGinnis et al., 1992).

Arrestin exhibits high-affinity binding to light-activated, phosphorylated rhodopsin ( $K_{\mathrm{D}}$ of $20 \mathrm{~nm}$ ) (Pulvermuller et al.,
1997), a lesser but detectable affinity for phosphorylated opsin (Gurevich and Benovic, 1993), and no measurable binding to light-activated, unphosphorylated rhodopsin in vitro. In vivo, the absence of rhodopsin phosphorylation results in abnormally prolonged, steplike single photon responses with a mean duration of $5 \mathrm{sec}$, indicating that arrestin does not bind to unphosphorylated rhodopsin within the time course of normal responses ( $<500 \mathrm{msec})$ (Chen et al., 1995, 1999a; Mendez et al., 2000). In fact, at least three phosphorylation sites appear to be required to trigger the conformational change in arrestin that allows it to bind to phosphorylated photolyzed rhodopsin under physiological conditions (Mendez et al., $2000)$. If generation of the light-activated, phosphorylated rhodopsin is the driving force behind arrestin movement, arrestin will fail to translocate or will translocate with much slower kinetics when rhodopsin phosphorylation is prevented during light exposure. However, in $\mathrm{CSM} / \mathrm{rho}^{-1-}$ and $\mathrm{RK}^{-1-}$ retinas, arrestin translocated in response to light to a similar extent and with a similar time course as the wild-type control (Fig. 1). This result shows that light-induced arrestin translocation occurs independently of the generation of its high-affinity-binding molecular target.

Transducin signaling is not required for light-driven arrestin translocation

Transducin is the only G-protein involved in phototransduction signaling in vertebrate rod photoreceptors. In the absence of transducin, retinal rods maintain their structure, but they do not respond to light (Calvert et al., 2000). Light-dependent arrestin translocation was investigated in mice lacking the transducin $\alpha$ subunit $\left(\operatorname{Tr}_{\alpha}{ }^{-l-}\right)$ to determine whether transducin signaling is required for arrestin movement (Fig. 2). As can be seen in Figure $2 A$, arrestin translocates with the same time course in control and $\operatorname{Tr}_{\alpha}{ }^{-1-}$ retinas. This result excludes phototransduction as the signal for arrestin movement during light exposure. Furthermore, it excludes the possibility that transducin may signal to motor proteins to promote arrestin movement.

In the absence of transducin, light-dependent rhodopsin phosphorylation is likely to proceed normally and could potentially serve as a sink for arrestin in the outer segment membranes. To rule out any possible redundancy between the phosphorylation of light-activated rhodopsin and transducin signaling in causing arrestin redistribution, arrestin localization was analyzed in $\operatorname{Tr}_{\alpha}{ }^{-1-} / \mathrm{RK}^{-1-}$ retinas. In response to light, arrestin immunoreactivity shifted to the outer segments in $\mathrm{Tr}_{\alpha}{ }^{-1-} / \mathrm{RK}^{-1-}$ retinas with a time course similar to that seen in wild-type control retinas (Fig. $2 B$ ), indicating that the mechanism for arrestin translocation is independent of rhodopsin phosphorylation and transducin signaling and/or translocation. 

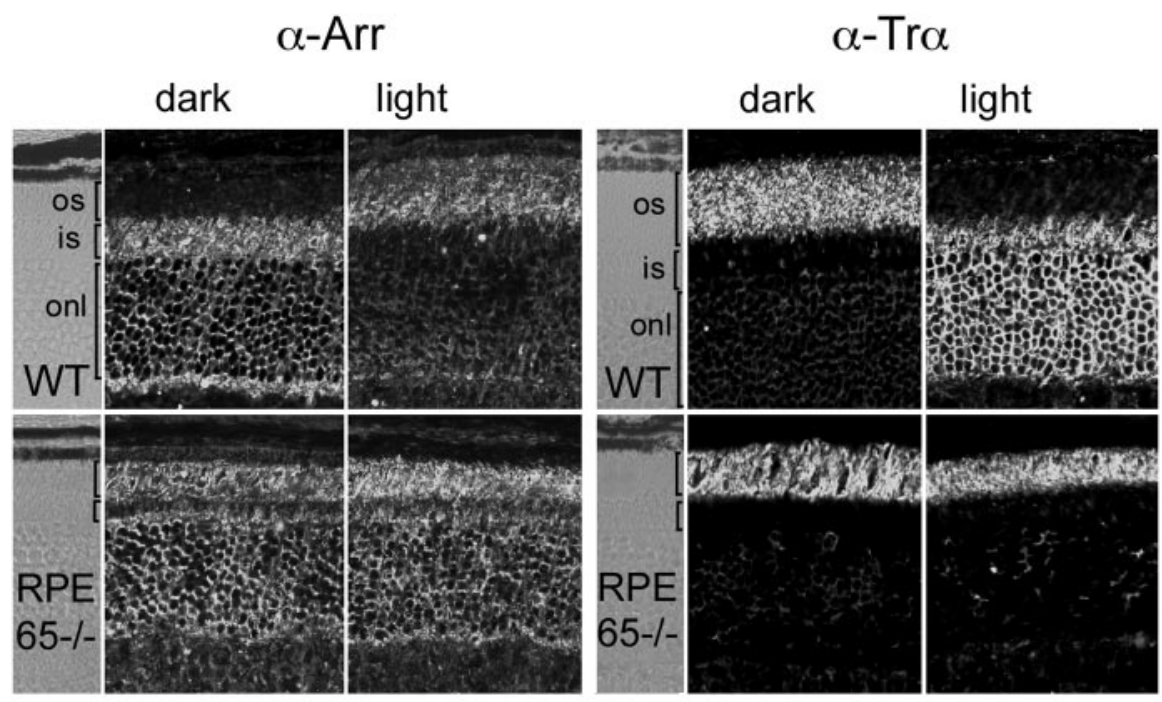

$70 \mu \mathrm{m}$

Figure 3. Light-independent arrestin and $\operatorname{Tr}_{\alpha}$ localization in RPE65 ${ }^{-1-}$ mice. Retinal sections $(10 \mu \mathrm{m})$ were obtained from 1-month-old RPE65 ${ }^{-1-}$ mice or wild-type controls (WT), either dark-adapted or exposed to 2000 lux white light for $20 \mathrm{~min}$ Arrestin immunoreactivity in dark-adapted wild-type mice is confined to the outer nuclear and inner segment layers, whereas in dark-adapted RPE65 ${ }^{-1-}$ mice, substantial immunostaining is present at the outer segments as well. Light had no effect on arrestin localization in RPE65 ${ }^{-1-}$ retinas (left). $\operatorname{Tr}_{\alpha}$ immunoreactivity was examined in sections adjacent to those used for arrestin (right). $\mathrm{Tr}_{\alpha}$ localization was restricted to the outer segments in RPE $65^{-1-}$ mice, independent of the lighting conditions. Similar results were obtained with two independent groups of mice. os, Outer segment; is, inner segment; onl, outer nuclear layer. even at the electron microscopy level, because of the presence of opsin (Redmond et al., 1998).

If arrestin translocation requires light activation of rhodopsin, no light-dependent arrestin translocation should be observed in these mice. As shown in Figure 3, arrestin immunostaining in RPE65 ${ }^{-1-}$ retinas was indistinguishable in dark- or light-adapted retinas. The pattern of arrestin immunoreactivity, however, differed from that of dark-adapted wild-type retinas (Fig. 3). In RPE $65^{-l-}$ retinas, arrestin immunolocalized substantially to the outer segments and to the outer nuclear layer and inner segments. This result indicates that opsin alone, without the retinal chromophore, has some signaling capacity to trigger arrestin translocation. Opsin has been reported to have a small but measurable catalytic activity in vitro (Robinson et al., 1992; Surya et al., 1995; Buczylko et al., 1996). This weak constitutive activity, however, is apparently not sufficient to trigger $\operatorname{Tr}_{\alpha}$ translocation to proximal compartments in $\mathrm{RPE}^{-1-}$ mice, because $\operatorname{Tr}_{\alpha}$ immunoreactivity is restricted to the outer segments in both darkand light-adapted retinas (Fig. 3). Therefore, opsin causes a partial translocation of arrestin by a mechanism that is independent of transducin signaling
and/or translocation, which is consistent with our previous results. This gain-of-function phenotype in RPE65 ${ }^{-1-}$ mice by the presence of opsin suggests that the light-dependent arrestin translocation is normally initiated by rhodopsin activation.

\section{Arrestin and transducin translocation in retinal rods are independent processes}

Light triggers a massive movement of transducin to the rod inner segment in the opposite direction from arrestin (Brann and Cohen, 1987; Philp et al., 1987; Whelan and McGinnis, 1988; Organisciak et al., 1991; McGinnis et al., 1992; Sokolov et al., 2002). We showed that arrestin translocation was unaffected in the absence of $\operatorname{Tr}_{\alpha}$. Therefore, we concluded that transducin is not required for arrestin movement. To determine whether transducin translocation is coupled to arrestin movement, transducin redistribution during light exposure was examined in retinas lacking arrestin (Xu et al., 1997). Figure 4 shows that, in the absence of arrestin, $\operatorname{Tr}_{\alpha}$ translocates normally and on a time course similar to that of wild-type control retinas.

From these results, we conclude that the translocation of arrestin and transducin in opposite directions during light exposure is not coupled and is likely to result from two independent mechanisms.

\section{Discussion}

In the present study, we used a genetic approach to demonstrate that neither phosphorylation of photolyzed rhodopsin nor transducin signaling is the biochemical basis behind light-dependent arrestin movement. Furthermore, we show that the lightregulation of arrestin movement is lost in the absence of rhodop$\sin$. Together, these results point to rhodopsin-mediated arrestin translocation through a new signaling pathway. 
That rhodopsin phosphorylation is not required for lightdriven translocation of arrestin in vivo eliminates passive diffusion to its high-affinity molecular target as the mechanism for arrestin movement, a hypothesis supported by previous studies in cultured retinas (Mangini and Pepperberg, 1988; Mangini et al., 1994). One mechanism by which arrestin redistribution in vertebrate rods could occur is by active transport along the microtubules present in the ciliary axoneme and along the rod outer segment incisures. These structures show immunoreactivity to the kinesin heavy chain (Eckmiller and Toman, 1998), a plus-end directed molecular motor. Interestingly, arrestin immunoreactivity accumulates at these structures in Xenopus retinas during dark adaptation, suggesting that arrestin is a cargo for microtubule-based transport after changes in lighting conditions (McGinnis et al., 2002). Consistent with the role of microtubular tracks in arrestin transport, arrestin was observed to accumulate at the inner segments of rods after conditional inactivation of the KIF3A subunit of kinesin II (Marszalek et al., 2000). However, future studies will be needed to investigate the nature of the interaction of arrestin with motor proteins or other components of the polarized transport machinery.

In vertebrate rod photoreceptors, transducin is the only G-protein known to be involved in phototransduction. In its absence, rod photoreceptors cannot respond to light (Calvert et al., 2000). However, we show that transducin is dispensable for light-triggered arrestin translocation. This result excludes both phototransduction and direct transducin signaling to the transport machinery as the trigger for arrestin movement. Although transducin is not required, results from the RPE $65^{-1-}$ mice point to rhodopsin as the light receptor for arrestin transport. Rhodopsin is primarily absent in RPE $65^{-1-}$ mice because of a defect in the visual cycle to regenerate 11-cis-retinal, but the presence of the opsin apoprotein preserves rod outer segment structure (Redmond et al., 1998). Opsin in RPE65 ${ }^{-1-}$ mice is constitutively phosphorylated (Ablonczy et al., 2002; Van Hooser et al., 2002), probably as a consequence of the weak constitutive activity of opsin described in vitro (Robinson et al., 1992; Surya et al., 1995; Buczylko et al., 1996). In RPE65 ${ }^{-1-}$ mice, opsin appeared to signal constitutively for arrestin translocation to rod outer segments, regardless of the lighting conditions. However, opsindriven arrestin translocation was only partial and was not completed by light exposure (Fig. 3). The finding that light had no effect on the arrestin immunolocalization pattern of RPE65 ${ }^{-1-}$ retinas excludes the involvement of other light receptors present in other retinal cell populations (Hattar et al., 2002) in signaling arrestin translocation.

$\operatorname{Tr}_{\alpha}$ immunoreactivity was restricted to rod outer segments, regardless of the lighting conditions in RPE65 ${ }^{-1-}$ retinas, suggesting that the weak catalytic activity of opsin in these mice was not sufficient to trigger detectable transducin movement. This might not be surprising given that opsin triggers excitation with a $10^{6}-10^{7}$ times smaller efficiency than rhodopsin (Cornwall and Fain, 1994) and given that the light intensity threshold for detection of transducin movement after $1 \mathrm{hr}$ of exposure was estimated to be 20 scotopic $\mathrm{cd} / \mathrm{m}^{2}$, resulting in the bleaching of $\sim 0.02 \%$ of the rhodopsin content $\left(\sim 10^{4}\right.$ rhodopsin molecules) per second (Sokolov et al., 2002). The restriction of $\operatorname{Tr}_{\alpha}$ immunolocalization to the rod outer segments of RPE $65^{-1-}$ retinas excluded the nonspecific mislocalization of proteins in these rods attributable to cytoskeletal disruption. Together, these results implicate rhodopsin as the light receptor for triggering both transducin and arrestin movement and point to a different requirement of the catalytic activity of rhodopsin for these independent processes.
The ratio of arrestin to rhodopsin in vertebrate rod outer segments has been estimated at 1:30 (Hamm and Bownds, 1986; Pugh and Lamb, 1990). Under illumination, this ratio may vary up to threefold because of light-triggered arrestin mobilization (Whelan and McGinnis, 1988). Within the physiological range of the response of the rod ( $<1000$ photoisomerizations/second), the concentration of arrestin in the outer segment is not limiting (Xu et al., 1997). Therefore, it is unlikely that arrestin translocation to the outer segment serves to regulate the rod photoresponse in the range of light intensities in which the rods are operational. Nevertheless, timely deactivation of excess photolyzed, phosphorylated rhodopsin is important for dark adaptation as the rod regains its dark current after bright light exposure. In addition, arrestin mobilization may dampen constitutive signaling resulting from excess photolyzed, phosphorylated rhodopsin generated during bright light exposures that would otherwise lead to cell death (Chen et al., 1999b).

How does rhodopsin signal arrestin translocation? Although no GTP-binding protein can substitute for transducin phototransduction signaling in vertebrate rods (Calvert et al., 2000), other G-proteins, such as $G_{\alpha q}$ and $G_{\alpha 11}$, appear to be present (Peng et al., 1997), and could couple to rhodopsin for other functions (Kasahara et al., 2002). We found that light-driven arrestin translocation is not affected in $\mathrm{G}_{\alpha 11}{ }^{-1-}$ mice or in $\mathrm{G}_{\alpha \mathrm{q}}{ }^{-1-}$ mice (A. Mendez, M. I. Simon, J. Chen, unpublished results), but their functional redundancy in other tissues (Offermanns, 2001) does not allow us to rule out their involvement in this process. A challenge for future studies will be to elucidate the molecular basis for this signaling pathway.

\section{References}

Ablonczy Z, Crouch RK, Goletz PW, Redmond TM, Knapp DR, Ma JX, Rohrer B (2002) 11-cis-retinal reduces constitutive opsin phosphorylation and improves quantum catch in retinoid-deficient mouse rod photoreceptors. J Biol Chem 277:40491-40498.

Arshavsky VY, Lamb TD, Pugh Jr EN (2002) G Proteins and phototransduction. Annu Rev Physiol 64:153-187.

Brann MR, Cohen LV (1987) Diurnal expression of transducin mRNA and translocation of transducin in rods of rat retina. Science 235:585-587.

Broekhuyse RM, Tolhuizen EFJ, Janssen APM, Winkens HJ (1985) Light induced shift and binding of S-antigen in retinal rods. Curr Eye Res 4:613-618.

Broekhuyse RM, Janssen APM, Tolhuizen EFJ (1987) Effect of light adaptation on the binding of $48-\mathrm{kDa}$ protein (S-antigen) to photoreceptor cell membranes. Curr Eye Res 6:607-610.

Buczylko J, Saari JC, Crouch RK, Palczewski K (1996) Mechanisms of opsin activation. J Biol Chem 271:20621-20630.

Burns ME, Baylor DA (2001) Activation, deactivation, and adaptation in vertebrate photoreceptor cells. Annu Rev Neurosci 24:779-805.

Calvert PD, Krasnoperova NV, Lyubarsky AL, Isayama T, Nicolo M, Kosaras B, Wong G, Gannon KS, Margolskee RF, Sidman RL, Pugh Jr EN, Makino CL, Lem J (2000) Phototransduction in transgenic mice after targeted deletion of the rod transducin alpha-subunit. Proc Natl Acad Sci USA 97:13913-13918.

Chen J, Makino CL, Peachey NS, Baylor DA, Simon MI (1995) Mechanisms of rhodopsin inactivation in vivo as revealed by a $\mathrm{COOH}$-terminal truncation mutant. Science 267:374-377.

Chen CK, Burns ME, Spencer M, Niemi GA, Chen J, Hurley JB, Baylor DA, Simon MI (1999a) Abnormal photoresponses and light-induced apoptosis in rods lacking rhodopsin kinase. Proc Natl Acad Sci USA 96:3718-3722.

Chen J, Simon MI, Matthes MT, Yasumura D, LaVail MM (1999b) Increased susceptibility to light damage in an arrestin knockout mouse model of Oguchi disease (stationary night blindness). Invest Ophthalmol Vis Sci 40:2978-2982.

Cornwall MC, Fain GL (1994) Bleached pigment activates transduction in isolated rods of the salamander retina. J Physiol (Lond) 480:261-279.

Eckmiller MS, Toman A (1998) Association of kinesin with microtubules in 
diverse cytoskeletal systems in the outer segments of rods and cones. Acta Anat 162:133-141.

Fain GL, Matthews HR, Cornwall MC, Koutalos Y (2001) Adaptation in vertebrate photoreceptors. Physiol Rev 81:117-151.

Gurevich VV, Benovic JL (1993) Visual arrestin interaction with rhodopsin. Sequential multisite binding ensures strict selectivity toward lightactivated phosphorylated rhodopsin. J Biol Chem 268:11628-11638.

Hamm HE, Bownds MD (1986) Protein complement of rod outer segments of frog retina. Biochemistry 25:4512-4523.

Hattar S, Liao HW, Takao M, Berson DM, Yau KW (2002) Melanopsincontaining retinal ganglion cells: architecture, projections, and intrinsic photosensitivity. Science 295:1065-1070.

Kasahara T, Okano T, Haga T, Fukada Y (2002) Opsin- $\mathrm{G}_{11}$-mediated signaling pathway for photic entrainment of the chicken pineal circadian clock. J Neurosci 22:7321-7325.

Koutalos Y, Yau KW (1996) Regulation of sensitivity in vertebrate rod photoreceptors by calcium. Trends Neurosci 19:73-81.

Mangini NJ, Pepperberg DR (1988) Immunolocalization of 48K in rod photoreceptors. Light and ATP increase OS labeling. Invest Ophthalmol Vis Sci 29:1221-1234.

Mangini NJ, Garner GL, Okajima TI, Donoso LA, Pepperberg DR (1994) Effect of hydroxylamine on the subcellular distribution of arrestin (Santigen) in rod photoreceptors. Vis Neurosci 11:561-568.

Marszalek JR, Liu X, Roberts EA, Chui D, Marth JD, Williams DS, Goldstein LSB (2000) Genetic evidence for selective transport of opsin and arrestin by kinesin-II in mammalian photoreceptors. Cell 102:175-187.

McGinnis JF, Whelan JP, Donoso LA (1992) Transient, cyclic changes in mouse visual cell gene products during the light-dark cycle. J Neurosci Res 31:584-590.

McGinnis JF, Matsumoto B, Whelan JP, Cao W (2002) Cytoskeleton participation in subcellular trafficking of signal transduction proteins in rod photoreceptor cells. J Neurosci Res 67:290-297.

Mendez A, Burns ME, Roca A, Lem J, Wu LW, Simon MI, Baylor DA, Chen J (2000) Rapid and reproducible deactivation of rhodopsin requires multiple phosphorylation sites. Neuron 28:153-164.

Offermanns S (2001) In vivo functions of heterotrimeric G-proteins: studies in $\mathrm{G} \alpha$-deficient mice. Oncogene 20:1635-1642.

Organisciak DT, Xie A, Wang HM, Jiang YL, Darrow RM, Donoso LA (1991) Adaptive changes in visual cell transduction protein levels: effect of light. Exp Eye Res 53:773-779.

Peng Y-W, Rhee SG, Yu W-P, Ho Y-K, Schoen T, Chader GJ, Yau K-W (1997) Identification of components of a phosphoinositide signaling pathway in retinal rod outer segments. Proc Natl Acad Sci USA 94:1995-2000.
Peterson JJ, Tam BM, Moritz OL, Shelamer CL, Dugger DR, McDowell JH, Hargrave PA, Papermaster DS, Smith WC (2003) Arrestin migrates in photoreceptors in response to light: a study of arrestin localization using an arrestin-GFP fusion protein in transgenic frogs. Exp Eye Res, in press.

Philp NJ, Chang W, Long K (1987) Light-stimulated protein movement in rod photoreceptor cells of the rat retina. FEBS Lett 225:127-132.

Pugh Jr EN, Lamb TD (1990) Cyclic GMP and calcium: the internal messengers of excitation and adaptation in vertebrate photoreceptors. Vision Res 30:1923-1948.

Pugh Jr EN, Nikonov S, Lamb TD (1999) Molecular mechanisms of vertebrate photoreceptor light adaptation. Curr Opin Neurobiol 9:410-418.

Pulvermuller A, Maretzki D, Rudnicka-Nawrot M, Smith WC, Palczewski K, Hofmann KP (1997) Functional differences in the interaction of arrestin and its splice variant, p44, with rhodopsin. Biochemistry 36:9253-9260.

Raport CJ, Lem J, Makino C, Chen CK, Fitch CL, Hobson A, Baylor D, Simon MI, Hurley JB (1994) Downregulation of cGMP phosphodiesterase induced by expression of GTPase-deficient cone transducin in mouse rod photoreceptors. Invest Ophthalmol Vis Sci 35:2932-2947.

Redmond TM, Yu S, Lee E, Bok D, Hamasaki D, Chen N, Goletz P, Ma JX, Crouch RK, Pfeifer K (1998) Rpe65 is necessary for production of 11cis-vitamin A in the retinal visual cycle. Nat Genet 20:344-351.

Robinson PR, Cohen GB, Zhukovsky EA, Oprian DD (1992) Constitutively active mutants of rhodopsin. Neuron 9:719-725.

Sokolov M, Lyubarsky AL, Strissel KJ, Savchenko AB, Govardovskii VI, Pugh Jr EN, Arshavsky VY (2002) Massive light-driven translocation of transducin between the two major compartments of rod cells: a novel mechanism of light adaptation. Neuron 34:95-106

Surya A, Foster KW, Knox BE (1995) Transducin activation by the bovine opsin apoprotein. J Biol Chem 270:5024-5031.

Van Hooser JP, Liang Y, Maeda T, Kuksa V, Jang G-F, He Y-G, Rieke F, Fong HKW, Detwiler PB, Palczewski K (2002) Recovery of visual functions in a mouse model of Leber congenital amaurosis. J Biol Chem 277:19173-19182.

Whelan JP, McGinnis JF (1988) Light-dependent subcellular movement of photoreceptor proteins. J Neurosci Res 20:263-270.

Wilden U (1995) Duration and amplitude of the light-induced cGMP hydrolysis in vertebrate photoreceptors are regulated by multiple phosphorylation of rhodopsin and by arrestin binding. Biochemistry 34:1446-1454 .

Wilden U, Hall SW, Kühn H (1986) Phosphodiesterase activation by photoexcited rhodopsin is quenched when rhodopsin is phosphorylated and binds the intrinsic $48-\mathrm{kD}$ a protein of rod outer segments. Proc Natl Acad Sci USA 83:1174-1178.

Xu J, Dodd RL, Makino CL, Simon MI, Baylor DA, Chen J (1997) Prolonged photoresponses in transgenic mouse rods lacking arrestin. Nature 389: 505-509. 